Abstract

\title{
Noncapped Genomic RNA Are Critical for Alphaviral Infection and Pathogenicity ${ }^{\dagger}$
}

\author{
Autumn T. LaPointe ${ }^{1,2}$ and Kevin J Sokoloski ${ }^{1,2, *}$ \\ 1 Department of Microbiology and Immunology, University of Louisville School of Medicine, \\ Louisville, KY 40202, USA; altown02@louisville.edu \\ 2 Center for Predictive Medicine and Emerging Infectious Diseases, University of Louisville, \\ Louisville, KY 40202, USA \\ * Correspondence: kevin.sokoloski@louisville.edu; Tel.: +1-(502)-852-2149 \\ † Presented at Viruses 2020-Novel Concepts in Virology, Barcelona, Spain, 5-7 February 2020. \\ Published: 13 June 2020
}

\begin{abstract}
Alphaviruses are positive-sense RNA arthropod-borne viruses that represent a significant threat to public health. During alphaviral replication, significant quantities of viral genomic RNAs that lack a canonical 5' cap structure are produced and packaged into viral particles, despite the fact that the noncapped genomes cannot be translated and are essentially noninfectious. Previously, we have reported that the capping efficiency of nsP1, the alphaviral capping enzyme, of Sindbis virus (SINV) could be modulated via point mutation. It was found that increasing RNA capping efficiency led to decreased viral growth kinetics via decreased particle production, despite increased innate immune evasion, whereas decreasing capping efficiency led to wild-type growth kinetics and particle production. This led to the conclusion that the noncapped viral RNAs meaningfully contribute to the biology of alphaviral infections at the molecular level. To determine the importance of the noncapped viral RNAs in vivo, we characterized the impact of altered capping efficiency in a murine model of infection utilizing a neurovirulent strain of SINV. Mice infected with the nsP1 mutant with decreased capping exhibited wild-type rates of mortality, weight loss, and neurological symptoms. Conversely, the mice infected with the increased capping nsP1 mutant showed significantly reduced mortality and morbidity compared to mice infected with the wild-type virus. Interestingly, viral titers in the ankle, serum, and brain were equivalent between the wild-type virus and the two mutant viruses. Importantly, examination of the brain tissue revealed that mice infected with the increased capping mutant had significantly reduced immune cell infiltration and expression of proinflammatory cytokines compared to the decreased capping mutant and wild-type virus. Collectively, these data indicate that the noncapped viral RNAs have important roles during the early and late stages of alphaviral infection and suggest a novel mechanism by which noncapped viral RNA aids in viral pathogenesis.
\end{abstract}

Keywords: RNA virus; alphavirus; capping efficiency; nsP1; pathogenesis

(C) 2020 by the authors. Licensee MDPI, Basel, Switzerland. This article is an open access article distributed under the terms and conditions of the Creative Commons Attribution (CC BY) license (http://creativecommons.org/licenses/by/4.0/). 